\title{
Grafting a Safeguard against Cocaine Abuse
}

\author{
MingXu ${ }^{1 *}$ and Xiaoyang $\mathrm{Wu}^{2 *}$ \\ ${ }^{1}$ Department of Anesthesia \&Critical Care, The University of Chicago, 5841 S. Maryland Avenue, Chicago, IL 60637, USA \\ ${ }^{2}$ Ben May Department for Cancer Research, The University of Chicago, 929 E 57thStreet, Chicago, IL 60637, USA \\ Address correspondence to Ming Xu,MXu@dacc.uchicago.edu, or XiaoyangWu,Xiaoyangwu@uchicago.edu
}

Received 14 September 2018; Revised 27 November 2018; Accepted 30 November 2018

Copyright (C) 2018 Ming Xu, et al. This is an open access article distributed under the terms of the Creative Commons Attribution License, which permits unrestricted use, distribution, and reproduction in any medium, provided the original work is properly cited.

Drug addiction is a brain disease characterized by compulsive drug-seeking and taking and a high likelihood of relapse when exposed to drugs or drugassociated cues, even long after abstention [1]. Relapse is the major challenge for the treatment of drug addiction in humans. Cocaine is a widely abused drug that causes significant morbidity and mortality. There are currently no FDA-approved medications for reducing cocaine use or treating relapse in cocaine addicts. Moreover, there are no interventions for the acute emergencies that result from cocaine overdose. A novel skin stem cell-based gene therapy has recently been described to be effective in reducing core features of cocaine abuse in laboratory mice [2], offering hope of producing the first medication for treating cocaine abuse, and adding a new tool for treating drug abuse and co-abuse.

The development of the cutaneous and gene-based safeguard against cocaine abuse was fueled by three technical advances. The first is the availability of the modified human butyrylcholinesterase (hBChE) [3]. $\mathrm{BChE}$ is a natural enzyme that is present in hepatocytes and plasma and it hydrolyzes its normal substrate acetylcholine. BChE can also hydrolyze cocaine into products low in toxicity and rewarding properties [3]. Recent advance in protein engineering has greatly enhanced catalytic potency and substrate specificity of $\mathrm{BChE}$ for cocaine hydrolysis [4], and for protecting against cocaine-induced behaviors, including acquisition and reinstatement of intravenous self-administration in rodents [5-6]. hBChE has a much higher catalytic efficiency than parent $\mathrm{hBChE}$ with significantly reduced activity for acetylcholine [4]. hBChE has a very short half-life in vivo, however, limiting its potential in treating cocaine abuse in users [7]. The second is the availability of the CRISPR technology which allows site-specific modification of target genomes with great precision [8], efficiency and reduced potential risks, offering an ideal alternative to the current delivery approaches with potential limitations and side-effects.

The third and perhaps the most important advance is that $\mathrm{Wu}$ and colleagues developed a method for preparation of mouse skin organoid culture and engraftment of the cultured mouse skin substitute to the immunocompetent host mice [2,9]. The epidermal progenitor cells of the skin have several major and unique advantages making them particularly suited for gene therapy ex vivo: i) skin is the most accessible organ in the body, offering availability for collection of epidermal progenitor cells with well-established procedures. It is easy to monitor the skin for potential off-target effects of gene targeting and to remove or replace it in case of adverse consequences. ii) Cultured epidermal progenitor cells can be induced to differentiate and the resultant cultured epidermal autograft (CEA) can be transplanted to donors with routine protocols. The grafted CEAs are stable in vivo and can last for decades in clinical followup studies. iii) Somatic gene therapy with epidermal progenitor cells is tissue specific. iv) Epidermal progenitor cells can withstand long-term culture in vitro without losing stemness, permitting engineering with genome editing technology using a viral-free approach. v) Epidermal progenitor cells have low immunogenicity. vi) It has been well-documented that proteins secreted by skin epidermal cells, can cross the epidermal/dermal barrier and reach circulation to achieve therapeutic effect in a systemic manner.

To address the urgent need to develop a long-lasting treatment of drug abuse, the $\mathrm{Wu}$ and $\mathrm{Xu}$ labs teamed up and tested whether a skin-based platform for longterm delivery of genes may provide a safeguard against 
and effective way of treating drug abuse in mice. They found that, upon targeting an hBChE gene into epidermal progenitor cells and grafting the engineered cells, the transgene products can be produced in vivo and can efficiently protect mice from cocaine-seeking behavior including acquisition and relapse, and cocaine overdose [2]. The approach is specific in protecting against cocaine abuse, and not abuse for methamphetamine and alcohol. Not surprisingly, expression of BChE in grafted mice removes cocaine much more quickly in vivo, leading to reduced extracellular DA and locomotor activity [2]. The implications of these results are potentially significant: i) cutaneous gene therapy through skin transplants that elicits drug elimination may offer a therapeutic option to address cocaine abuse, including reducing ongoing and continuous use, relapse induced by drugs, and lethality in users and addicts seeking help or treatment. ii) This approach is expected to be long-lasting, highly specific and efficient with little individual variation. iii) It is minimally invasive, not tumorigenic, safe with minimal immune responses, low maintenance and high compliance. iv) The cutaneous gene therapy can also be expanded to treat environmental cue- and stressinduced relapse, other drugs of abuse including alcohol, nicotine and opioid and co-abuse of different drugs, and other comorbid conditions. Although much work is still needed before clinical trials can begin, a very recent and encouraging clinical intervention showed that transgenic CEAs could be used to replace the entire skin epidermis and saved a pediatric patient from the life-threatening skin disease, junctional epidermolysis bullosa, strongly supporting the feasibility of the cutaneous gene therapy [10].

Available gene therapies involve complex procedures and are extremely expensive. For examples, Glybera, the first AAV-based therapy approved by EU for treatment of lipase deficiency, costs more than $\$ 1$ million dollars [11]. By contrast, isolation of skin stem cells, CRISPR targeting and preparation of genetically modified cultured epidermal autograft are well-established and routine technically. There are several commercial CEAs available $\left(\sim \$ 1,000\right.$ per $\left.100 \mathrm{~cm}^{2}\right)[12]$ and the grafting procedures are relatively inexpensive [13]. The cutaneous gene therapy involves grafting of immunocompetent host, does not require lengthy hospitalizations, and the grafting procedure has been clinically used for treating burn wounds for decades. Together, the use of skin epidermal progenitor cells to deliver one or more therapeutic agents has potential to be feasible and affordable for treating drug abuse in humans. These key features of the new approach make it very hopeful of providing a desirable new tool in the near future to help millions of people with drug misuse disorders.

\section{References}

1. G. F. Koob, and N. D. Volkow, Neurobiology of addiction: a neurocircuitry analysis, Lancet Psychiatry, 3(2016), 760-773.

2. Y.Li, Q. Kong, J. Yue, X. Gou, X. Ming, and W. Xiaoyang, Genomeedited skin epidermal stem cells protect mice from cocaine-seeking behavior and cocaine overdose, Nature BME, (2018) -018-0293-z.

3. O. Lockridge, Review of human butyrylcholinesterase structure, function, genetic variants, history of use in the clinic, and potential therapeutic uses, Pharmacol Ther, 148(2015), 34-46.

4. F. Zheng, L. Xue, S. Hou, J. Liu, Z. Max, Y. Wenchao, et al., A highly efficient cocaine-detoxifying enzyme obtained by computational design, Nat Commun, 5(2014), 3457

5. J. J. Anker, S. Brimijoin, Y. Gao, L. Geng, N. E. Zlebnik, R. J. Parks, et al., Cocaine hydrolase encoded in viral vector blocks the reinstatement of cocaine seeking in rats for 6 months, Biol Psychi 71(2012), 700-705.

6. N. E. Zlebnik, S. Brimijoin, Y. Gao, A. T. Saykao, R. J. Parks, and M. E. Carroll, Long-term reduction of cocaine self-administration in rats treated with adenoviral vector-delivered cocaine hydrolase: evidence for enzymatic activity. Neuropsychopharm, 39(2014), 1538-1546.

7. Y. Gilgun-Sherki, R. E. Eliaz, D. J. McCann, P. S. Loupe, E. Eyal, $\mathrm{K}$. Blatt, et al., Placebo-controlled evaluation of a bioengineered, cocaine-metabolizing fusion protein, TV-1380 (AlbuBChE), in the treatment of cocaine dependence, Drug Alcohol Depend, 166(2016), 13-20.

8. D. B. Cox, R. J. Platt, and F. Zhang, Therapeutic genome editing: prospects and challenges. Nat Med 21(2015), 121-131.

9. J. Yue, X. Gou, C. Li, B. Wicksteed, and X. Wu, Treatment of diabetes and obesity with CRISPR-mediated genome editing in epidermal progenitor cells, Cell Stem Cell, 21(2017), 256-263.

10. T. Hirsch, T. Rothoeft, N. Teig, J. W. Bauer, G. Pellegrini, L. D. Rosa, et al., Regeneration of the entire human epidermis using transgenic stem cells, Nature 551(2017), 327-332.

11. L. Naldini, Gene therapy returns to centre stage, Nature, 526(2015), 351-360.

12. I. Brockmann, J. Ehrenpfordt, T. Sturmheit, M. Brandenburger, C. Kruse, M. Zille, et al., Skin-Derived stem cells for wound treatment using cultured epidermal autografts: clinical applications and challenges, Stem Cells Int, 2018(2018), 4623615.

13. F. R. Pierce Jr, and K. A. Richardson, Cost comparison of split thickness skin grafting with and without bilayer dermal regenerative templates for bilateral axillary hidradenitis suppurativa: A retrospective comparison study, Wound Medicine, 7(2014), 18-23. 